\title{
Ex Vivo Myelination and Remyelination in Cerebellar Slice Cultures as a Quantitative Model for Developmental and Disease-Relevant Manipulations
}

\author{
Kimberle Shen ${ }^{1}$, Tracy J. Yuen ${ }^{1}$ \\ ${ }^{1}$ Department of Neuroscience, Genentech Inc.
}

\section{Corresponding Author}

Tracy J. Yuen

hugenberger.tracy@gene.com

\section{Citation}

Shen, K., Yuen, T.J. Ex Vivo Myelination and Remyelination in Cerebellar Slice Cultures as a Quantitative Model for Developmental and Disease-Relevant Manipulations. J. Vis. Exp. (160), e61044, doi:10.3791/61044 (2020).

\section{Date Published}

June 12,2020

DOI

$10.3791 / 61044$

URL

jove.com/video/61044

\section{Abstract}

Studying myelination in vitro and in vivo poses numerous challenges. The differentiation of oligodendrocyte precursor cells (OPCs) in vitro, although scalable, does not recapitulate axonal myelination. OPC-neuron cocultures and OPC-fiber cultures allow for the examination of in vitro myelination, but they lack additional cell types that are present in vivo, such as astrocytes and microglia. In vivo mouse models, however, are less amenable to chemical, environmental, and genetic manipulation and are much more labor intensive. Here, we describe an ex vivo mouse cerebellar slice culture (CSC) quantitative system that is useful for: 1) studying developmental myelination, 2) modeling demyelination and remyelination, and 3) conducting translational research. Sagittal sections of the cerebellum and hindbrain are isolated from postnatal day $(P)$ 0-2 mice, after which they myelinate ex vivo for 12 days. During this period, slices can be manipulated in various ways, including the addition of compounds to test for an effect on developmental myelination. In addition, tissue can be fixed for electron microscopy to assess myelin ultrastructure and compaction. To model disease, CSC can be subjected to acute hypoxia to induce hypomyelination. Demyelination in these explants can also be induced by lysolecithin, which allows for the identification of factors that promote remyelination. Aside from chemical and environmental modifications, CSC can be isolated from transgenic mice and are responsive to genetic manipulation induced with Ad-Cre adenoviruses and tamoxifen. Thus, cerebellar slice cultures are a fast, reproducible, and quantifiable model for recapitulating myelination.

\section{Introduction}

Myelination of axons enables the rapid propagation of action potentials, a mechanism known as saltatory conduction ${ }^{1}$.
The importance of myelin is underscored by demyelinating diseases, such as multiple sclerosis (MS), which include 
a wide range of debilitating manifestations, including loss of vision, cognitive issues, and paralysis. There is no cure for MS, and current therapies focus on limiting disease progression by targeting peripheral immune cells. Disability in MS and related diseases is thought to be driven by a failure of remyelination and progressive neurodegeneration. In particular, demyelination, atrophy, and axonal loss are observed in progressive $\mathrm{MS}^{2,3}$. Thus, promoting remyelination represents a promising strategy that could be carried out in parallel to current treatments and yield additional therapeutic benefits.

In the central nervous system (CNS), myelination is carried out by specialized glial cells known as oligodendrocytes. Oligodendrocyte precursor cells (OPCs) differentiate into mature, myelinating oligodendrocytes through a series of highly orchestrated steps, including the growth of processes that contact axons, increases in morphological complexity, expansion of the myelin membrane, and finally, myelin sheath compaction ${ }^{4}$. Thus, the interaction between oligodendrocytes and neurons is highly intimate. Reciprocal interactions between neurons and oligodendrocytes are also required for the health and maintenance of the $\mathrm{CNS}^{2}$. Axonal activity plays a role in stimulating myelination and glial neurotrophic factors support the integrity of neurons. The importance of glia-glia crosstalk in the CNS is also becoming increasingly recognized $5,6,7,8$. For instance, astrocytic factors can influence the differentiation of OPCs and the maintenance of white matter tracts. Microglia also play a role in modulating OPC differentiation as well as clearing myelin debris, an important step of the remyelination process. Identifying cell-autonomous factors and understanding the influence of other CNS cell types in demyelination and remyelination will be invaluable for developing therapies for demyelinating and dysmyelinating diseases.

Here, we describe an ex vivo system using mouse cerebellar slice cultures (CSC) that allows for the manipulation and quantification of intact CNS tissue. Use of CSC allows myelination, or remyelination after the induction of demyelination by lysolecithin ${ }^{9,10}$, to be measured using methods traditionally used with in vivo studies, such as immunostaining and electron microscopy ${ }^{11,12,13}$. Lysolecithin is a membrane-disrupting chemical that results in a rapid loss of myelin and oligodendrocytes. A potential caveat to note is that lysolecithin may also result in the reduction of other cell types close to the lesion area. Unlike in vivo experiments, however, sagittal slices of the cerebellum can be easily manipulated via the addition of compounds, or genetically altered using Ad-Cre adenoviruses. The method also allows for manipulation of isolated tissue from transgenic mice or mice subjected to environmental insults such as hypoxia $^{11}, 12,13,14$. The CSC model thus allows the study of developmental myelination, modeling of disease, and identification of factors that promote or inhibit myelination, while integrating the contributions of different CNS cell types to oligodendrocyte function.

\section{Protocol}

All animal studies were authorized and approved by the Genentech Institutional Animal Care and Use Committee.

\section{Preparation of media and supplies for dissection ( $30-45 \mathrm{~min})$}

1. Prepare and sterile filter slice culture medium (SCM) and dissection medium (DM) as detailed in Table 1. Store at $4{ }^{\circ} \mathrm{C}$. If any factors are being tested for the myelination assay, add them to the SCM just before use. 
2. Pipette $1 \mathrm{~mL}$ of SCM into each well of 6 well plate.

3. Using sterile forceps, place an organotypic insert into each well, ensuring no bubbles are trapped under the membrane. Warm the plate with media at $37{ }^{\circ} \mathrm{C}$ in $7.5 \%$ $\mathrm{CO}_{2}$ incubator.

\section{Preparation of the dissection area ( $\sim 5-10 \mathrm{~min})$}

1. Perform procedures in a sterile laminar flow hood or on the benchtop with adequate aseptic techniques for all steps of the procedure. Wipe all areas clean with $70 \%$ ethanol.

2. Place a new blade and silicone cutting stage on the tissue chopper. Pipette $\sim 300 \mu \mathrm{L}$ of sterile water or $70 \%$ ethanol under the cutting stage to ensure it stays in place.

3. Use a cotton swab to gently wipe the tissue chopper blade and cutting stage with $70 \%$ ethanol. Dry before using the tissue chopper.

4. Spray all dissection tools with $70 \%$ ethanol and dry prior to the dissection.

5. Prepare two $10 \mathrm{~cm}$ Petri dishes: one with $15-20 \mathrm{~mL}$ of $\mathrm{DM}$, one with $10 \mathrm{~mL}$ of SCM. Store on ice. Keep all media and Petri dishes on ice when not dissecting.

\section{Cerebellar slice culture dissection ( $15-20$ min per pup)}

1. Place the Petri dish with DM on a dissecting microscope.

2. Euthanize a mouse pup, postnatal day $\mathrm{PO}-2$, by swift decapitation with sharp scissors.

3. Place the head in the Petri dish with DM under the dissecting microscope to clear blood.

4. Using fine scissors, cut once on each lateral edge at the base of the skull.
5. With the head placed upside down in DM, press down firmly on the underside of the skull using $\# 5 / 45$ forceps, forcing the undamaged hindbrain through the hole in the skull.

6. Under the dissecting microscope, cut away excess tissue using two \#11 scalpels. Ensure the cerebellum remains attached to the underlying piece of hindbrain. See Figure 1A for a detailed diagram.

7. Using the spatula, transfer the cerebellum to the cutting stage of the tissue chopper with the tissue resting on the rostral face of the hindbrain and the caudal face of the hindbrain facing the researcher. Ensure that the medial plane of the brain is exactly parallel to the tissue chopper blade.

8. Using the P200 pipette, remove excess medium around the tissue, ensuring that it is still moist but not floating or surrounded by liquid.

9. Swap the Petri dish containing DM with the Petri dish containing SCM under the dissecting microscope.

10. Cut the tissue into $350 \mu \mathrm{m}$ thick slices using the tissue chopper.

NOTE: The blade speed (e.g., roughly one cut per second) and force need to be optimized to ensure optimal cutting conditions. In rare instances, the tissue will get caught on the blade. To minimize loss of tissue sections, keep the thumb on the power button to quickly turn off the tissue chopper if needed.

11. Gently pipette $100 \mu \mathrm{L}$ of SCM under the tissue, so the slices are floating. Transfer the sliced tissue using the spatula to the Petri dish with SCM under a dissecting microscope. 
12. Use a cotton swab to gently wipe the tissue chopper blade and cutting stage with $70 \%$ ethanol.

13. Using the scalpels (blunt side), gently separate the slices under the dissecting microscope. Using a scalpel and spatula, transfer slices to the membrane in the 6 well plate with SCM.

NOTE: The most medial sections will often have the best myelination and tissue integrity. On average, 4-6 slices can be dissected from one mouse brain.

14. Incubate slices in $\mathrm{SCM}$ at $37^{\circ} \mathrm{C}$ in $7.5 \% \mathrm{CO}_{2}$.

\section{Culture and media changes ( 15-30 min)}

1. Replace the SCM every other day.

2. Pipette $1 \mathrm{~mL}$ of fresh medium into each well of a new 6 well plate and warm in an incubator. Using sterile forceps, transfer membranes to the new plate, ensuring no bubbles are underneath. Return plates to the incubator.

\section{Standard myelination and remyelination}

1. Standard myelination protocol (Figure 1B).

1. Culture slices dissected in section 3 from $0-12$ days in vitro (DIV). If the effect of any factor on myelination is being tested, add it to the medium at the time of culturing (0 DIV) and replenish it with each media change.

2. Fix at 12 DIV (step 7.1), a timepoint optimized for incomplete myelination $11,12,13$. This allows for the detection of any enhancing or blocking effects on myelination due to the factor added to the medium. A representative positive result with the addition of 100 $\mathrm{ng} / \mathrm{mL}$ Activin $\mathrm{A}$ is shown in Figure 2.
2. Remyelination protocol (Figure 1C)

1. Follow the standard myelination protocol described in section 5.1 with no additional factors until 14 DIV, the timepoint when the slices are fully myelinated $^{11,12,13}$.

2. Prepare lysolecithin stock $(125 \mathrm{mg} / \mathrm{mL})$ as detailed in Table 1. Pipette $1 \mathrm{~mL}$ of $0.5 \%$ lysolecithin in SCM into each well of a new 6 well plate and warm in an incubator.

3. Transfer membranes to a lysolecithin plate and place in an incubator overnight for 16-18 h.

4. The next day, prepare new plates with fresh SCM, adding any factor(s) being tested for remyelination effects if needed. Warm plates in an incubator.

5. For the media change, gently tap the membrane on the side of the well to ensure all the lysolecithin medium is removed. Replenish the factor(s) being tested at each media change (section 4).

6. Fix slices at 28 DIV (step 7.1), a timepoint optimized for incomplete remyelination. This allows for the detection of enhancement or blocking of remyelination due to the factor added to the medium. A representative positive result with the addition of 0.1 $\mu \mathrm{M}$ XAV939 is presented in Figure 3.

\section{Variations to the myelination and remyelination protocol}

1. Hypoxia-induced hypomyelination protocol (Figure 1D) ${ }^{11,13}$.

NOTE: This is presented as a variation of the standard myelination protocol presented in section 5.1.

1. Plate slices in SCM without factors. 
2. Transfer slice plates to a hypoxic incubator $\left(2 \% \mathrm{FiO}_{2}\right)$ for $24 \mathrm{~h}$ between 2-3 DIV.

3. The next day, prepare new plates with fresh SCM, adding any factor(s) being tested for myelination potential if needed. Warm plates in an incubator.

4. Following culture in hypoxia, change medium (section 4) and return cultures to a standard culture incubator $\left(37{ }^{\circ} \mathrm{C}, 7.5 \% \mathrm{CO}_{2}\right)$. Replenish any factors being tested with each subsequent media change (section 4).

5. Fix at 12 DIV (step 7.1).

2. Genetic manipulation protocol (Figure 1E) $)^{13}$

1. Isolate tissue from transgenic mice for dissection as in section 3 .

2. With inducible transgenic mouse lines, tamoxifen (100 $\mathrm{nM}$, a dose that does not induce myelination or OPC differentiation) or an Ad-Cre adenovirus can be added to SCM during media changes done at 1 DIV and 3 DIV.

3. Following the genetic manipulation with tamoxifen or Ad-Cre adenovirus, continue media changes every other day as in section 4 (with factors added if needed).

NOTES: All slices from one mouse will fit on one membrane, allowing for genotyping after dissection, thus making it simple to do experiments with transgenic mice.

\section{Tissue processing and analysis}

1. Fix slices by submerging the membrane gently in $4 \%$ paraformaldehyde for $1 \mathrm{~h}$ at room temperature (RT).
2. Rinse gently by submerging in phosphate buffered saline (PBS). Then, submerge in PBS in a 6 well plate.

NOTE: The protocol can be paused here. Fixed slices can be stored in PBS at $4{ }^{\circ} \mathrm{C}$ for up to a month. If not storing slices, rinse $2 x$ in PBS for 5 min before proceeding.

3. Use a scalpel to cut out the membrane from the ring and proceed with staining in a 6 well plate. Be sure to keep slices facing up (membrane-side down) for staining and subsequent mounting on slides.

4. Block for $1 \mathrm{~h}$ at $\mathrm{RT}$ in block solution (3\% heat-inactivated horse serum, $2 \%$ bovine serum albumin, and $0.25 \%$ Triton-X 100 diluted in PBS).

NOTE: If antigen retrieval is needed for antibody staining (e.g., CC1/Olig2), this can be done in glass Petri dishes.

5. Wrap plates in paraffin film and incubate overnight at $4{ }^{\circ} \mathrm{C}$ in primary antibody diluted in blocking solution.

6. Wash $3 x$ in blocking solution: 1) brief rinse, 2) $15 \mathrm{~min}$ wash, and 3) $1 \mathrm{~h}$ wash. For brief rinse, fill a small Petri dish with solution and gently submerge membrane using forceps before transferring to fresh solution. For longer washes (i.e., 15 min and $1 \mathrm{~h}$ ), submerge membrane in solution in a 6 well plate and place on a gentle rocker.

7. Incubate covered from light for $2 \mathrm{~h}$ at $\mathrm{RT}$ in secondary antibodies diluted 1:500 in PBS.

8. Wash 3x in PBS: 1) brief rinse, 2) 15 min wash, and 3) $1 \mathrm{~h}$ wash.

9. If DAPI staining is needed, submerge in 1:1,000 DAPI solution for $7 \mathrm{~min}$ at RT. Rinse briefly and then wash for 15 min in PBS.

10. Rinse in double-distilled water.

11. Mount membranes on a slide with slices facing up (membrane-side down). 
NOTE: Mounted slices can be stored at $4{ }^{\circ} \mathrm{C}$. Imaging on a confocal microscope is best done within 1 week, before the membrane becomes opaque and difficult to image.

12. Image slices using confocal microscopy and quantify as described previously $11,12,13$.

\section{Representative Results}

Cerebellar slice cultures derived from P0-2 mice (Figure 1A) were used to study myelination and assess the effects of the addition of various factors from 0-12 DIV (Figure 1B). To study remyelination, slice cultures at 14 DIV were first demyelinated with lysolecithin and allowed to remyelinate for 14 additional days in culture with the tested factors (Figure 1C), after which remyelination was quantified. The effect of hypoxia on myelination was also studied by placing slice cultures in a $2 \% \mathrm{FiO}_{2}$ hypoxic incubator for 24 h, from 2-3 DIV (Figure 1D). Lastly, slice cultures derived from transgenic mice were used to study the effect of gene knockouts on myelination. In this system, Cre recombination was induced by the addition of tamoxifen or Ad-Cre adenovirus at 1 DIV and 3 DIV, and slice cultures fixed for analysis at 12 DIV (Figure 1E). After fixation of tissues, quantification of demyelination and remyelination was carried out by immunostaining (Figure 2, Figure 3) and electron microscopy $11,12,13$.

Representative data shown (Figure 2, Figure 3) illustrate the dynamic range of myelination and remyelination in slice cultures. The myelination index was quantified by the ratio of Caspr, a paranodal marker that is an indirect readout of compact myelin, to neurofilament protein $\mathrm{H}(\mathrm{NFH})$, which stains axons. This myelination index has been validated to represent formation of compact myelin by electron microscopy and sodium channel staining ${ }^{12}$. Lysolecithininduced demyelination ${ }^{9,10}$ led to complete disappearance of Caspr-positive paranodes (myelination index $=0$, Figure 3A) and loss of compact myelin, which was restored during remyelination ${ }^{11,12}$.

To demonstrate a positive result on myelination, slices were cultured with Activin A following the timeline in Figure 1B. Activin A engages activin receptors on oligodendrocytes and drives oligodendrocyte differentiation and myelin compaction ${ }^{15}$. The representative data show that treating slices with $100 \mathrm{ng} / \mathrm{mL}$ of Activin A during myelination resulted in a higher myelination index (Figures 2A,B). Consistent with this, Activin A treatment accelerated OPC differentiation, as quantified by the ratio of CC1-positive mature oligodendrocytes to Olig2+ oligodendrocyte lineage cells $^{11,13}$ (Figures 2C,D).

A small molecule inhibitor of Tankyrase, XAV939, has been shown to promote myelination and remyelination by stabilization of Axin2 levels in oligodendrocytes ${ }^{11}$. Following lysolecithin treatment, demyelination was visualized and quantified in slice cultures by fragmented myelin basic protein (MBP) staining and lack of Caspr-positive paranodes (Figures 3A). Treatment with $0.1 \mu \mathrm{M}$ XAV939 during remyelination (15-28 DIV) significantly increased the myelination index compared to vehicle controls, as quantified by the ratio of Caspr to NFH staining (Figures 3B, C). The slice culture model can thus be used to examine and quantify effects on OPC differentiation, myelination, and remyelination in intact tissue. 

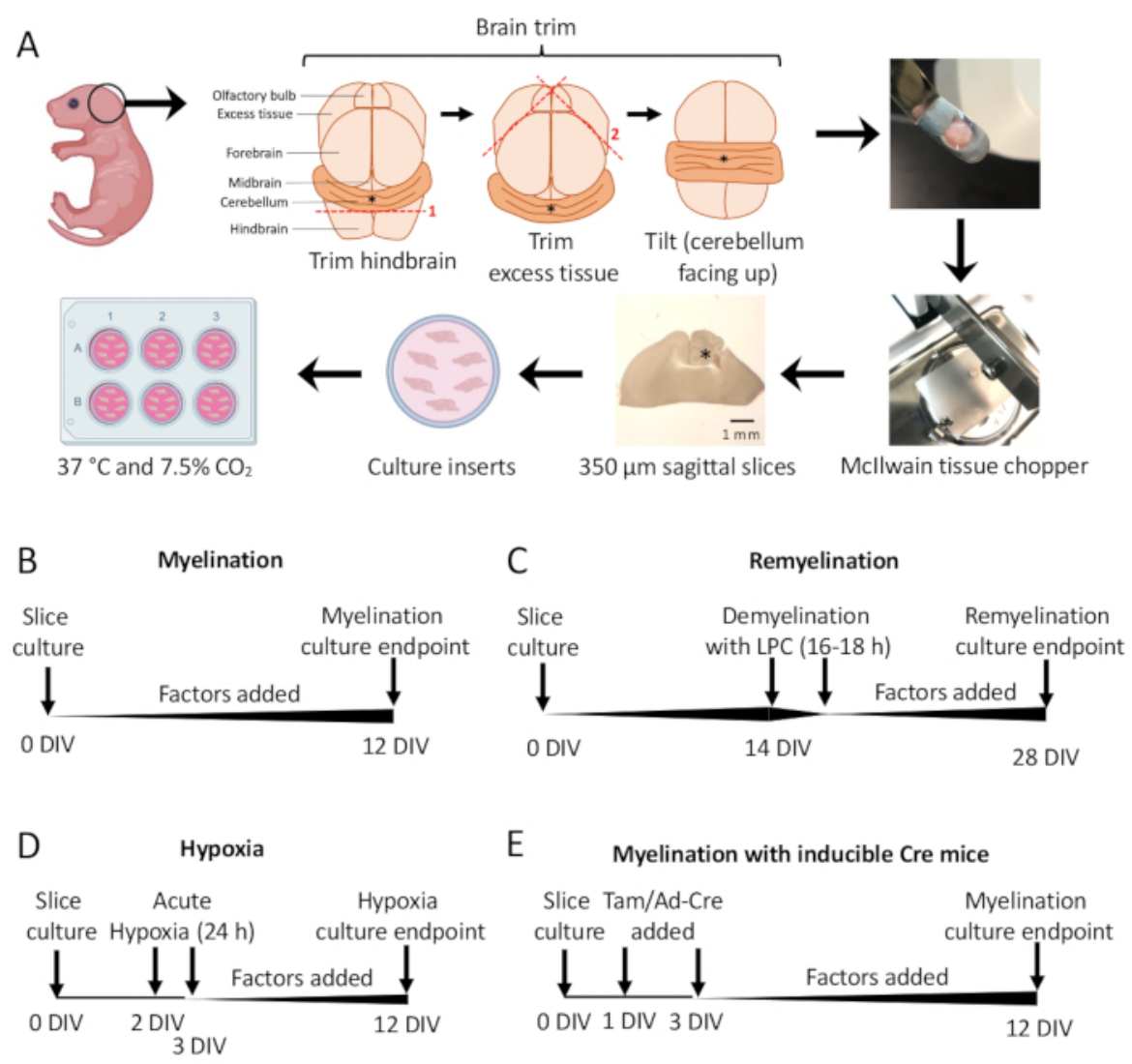

Figure 1: Schematic of cerebellar slice cultures showing key steps in the dissection process and timelines for different slice culture protocols. (A) Depiction of steps to generate slice cultures: dissection of brains from P0-2 mouse pups, trimming the brain (as shown), cutting the brain with a tissue chopper to generate $350 \mu \mathrm{m}$ sagittal slices, placing slices on organotypic culture inserts in a 6 well dish, and incubating at $37{ }^{\circ} \mathrm{C}$ and $7.5 \% \mathrm{CO}_{2} \cdot{ }^{*}$ denotes cerebellum. (B) Timeline for myelinating slice cultures. Slice cultures were incubated for 12 days before fixation for analysis. (C) Timeline for remyelinating slice cultures. Slices were treated with lysolecithin at 14 DIV for 16-18 $\mathrm{h}$ to induce complete demyelination and allowed to remyelinate until 28 DIV before fixation for analysis. (D) Timeline for myelination following hypoxic insult. Slices were exposed to an acute hypoxic insult for $24 \mathrm{~h}$ between 2-3 DIV, which caused hypomyelination. (E) Timeline for CSC from genetically modified mice. Tamoxifen (Tam, 100nM) or Ad-Cre virus was added at 1 DIV and 3 DIV to induce genetic modification, and slices were analyzed at 12 DIV. Timeline diagrams adapted from previous publications ${ }^{11,13}$. Please click here to view a larger version of this figure. 

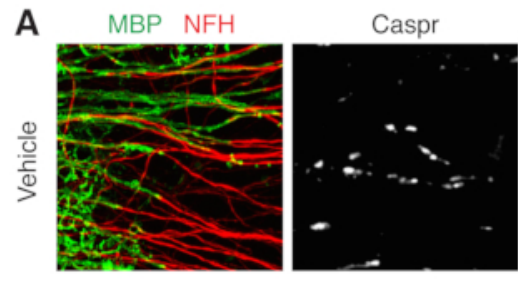

\section{B}
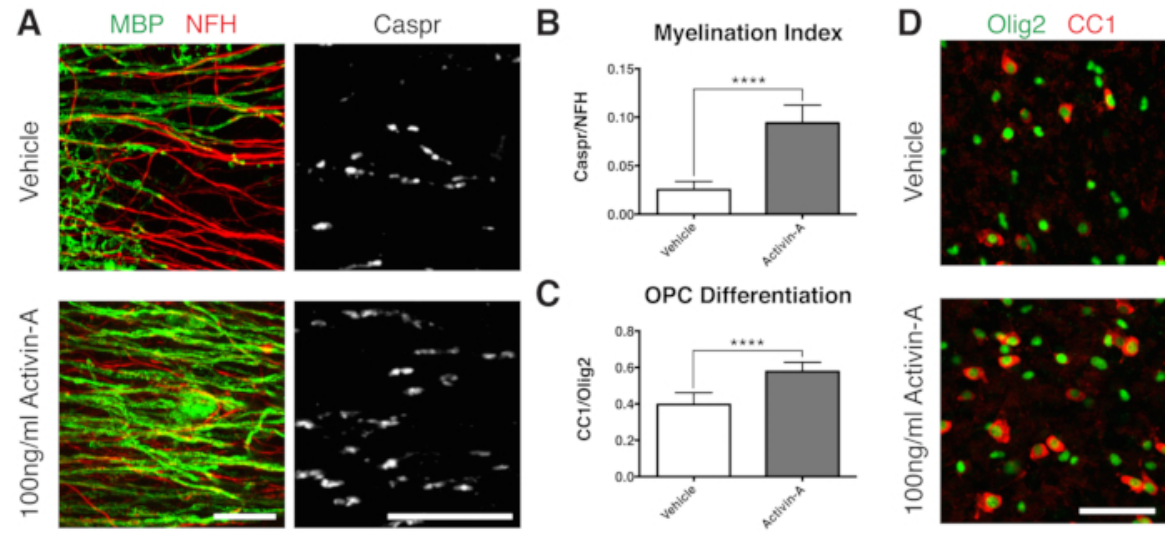

Figure 2: Representative data showing Activin A promotes myelination and OPC differentiation. (A) Increase in myelination (MBP) and paranodes (Caspr) with $100 \mathrm{ng} / \mathrm{mL}$ Activin A treatment, shown by immunostaining of slice cultures.

(B) Quantification of myelination index (ratio of area stained for Caspr+ paranodes to area stained for NFH+ axons).

(C) Quantification of OPC differentiation (ratio of CC1+ oligodendrocytes to Olig2+ oligodendrocyte lineage cells). (D)

Representative images of CC1/Olig2 staining. Values shown are mean + standard deviation. ${ }^{* * * *} \mathrm{P}<0.0001$; unpaired T-test. Scale bars: $A=25 \mu \mathrm{m}, \mathrm{D}=50 \mu \mathrm{m}$. Please click here to view a larger version of this figure.
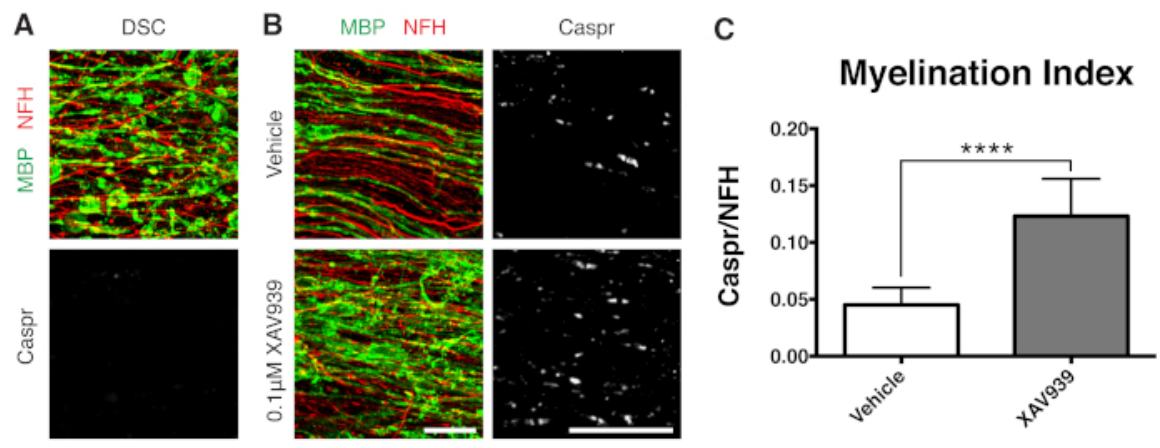

Figure 3: Representative data showing XAV939 promotes remyelination. (A) Images showing demyelination by fragmented MBP staining and lack of Caspr paranodes in slice cultures treated with lysolecithin. (B) Increase in remyelination (MBP) and paranodes (Caspr) with $0.1 \mu \mathrm{M}$ XAV939 treatment following lysolecithin-induced demyelination shown by immunostaining of slice cultures. (C) Quantification of myelination index (ratio of area stained for Caspr+ paranodes to area stained for $\mathrm{NFH}+$ axons). Values shown are mean + standard deviation. ${ }^{* * *} \mathrm{P}<0.0001$; unpaired T-test. Scale bars $=25 \mu \mathrm{m}$. Please click here to view a larger version of this figure. 


\begin{tabular}{|c|c|}
\hline Volume & Reagent \\
\hline $100 \mathrm{~mL}$ & Minimum Essential Media (MEM), HEPES, no glutamine \\
\hline $50 \mathrm{~mL}$ & Heat-inactivated horse serum \\
\hline $50 \mathrm{~mL}$ & Earle's balanced salt solution \\
\hline $2 \mathrm{~mL}$ & Penicillin-streptomycin 10,000 U/mL \\
\hline $2 \mathrm{~mL}$ & GlutaMAX supplement \\
\hline $2888 \mu \mathrm{L}$ & $45 \%$ glucose solution \\
\hline $1 \mathrm{~mL}$ & Fungizone \\
\hline \multicolumn{2}{|c|}{ Sterile filter through a $0.22 \mu \mathrm{m}$ filter and store in $4^{\circ} \mathrm{C}$ fridge for up to 2 weeks. } \\
\hline \multicolumn{2}{|c|}{ Dissection media (DM) } \\
\hline Volume & Reagent \\
\hline $100 \mathrm{~mL}$ & MEM, HEPES, with Earle's salts \\
\hline $1 \mathrm{~mL}$ & Penicillin-streptomycin 10,000 U/mL \\
\hline \multicolumn{2}{|c|}{ Sterile filter through a $0.22 \mu \mathrm{m}$ filter and store in $4{ }^{\circ} \mathrm{C}$ fridge for up to 2 months. } \\
\hline \multicolumn{2}{|c|}{ Lysolecithin stock (125 mg/mL) } \\
\hline Amount & Reagent \\
\hline $100 \mathrm{mg}$ & Lysolecithin \\
\hline $0.8 \mathrm{~mL}$ & Sterile PBS \\
\hline \multicolumn{2}{|c|}{ Dissolve $100 \mathrm{mg}$ of lysolecithin in $0.8 \mathrm{~mL}$ of sterile PBS. Store $80 \mu \mathrm{L}$ aliquots of stock solution at $-20^{\circ} \mathrm{C}$. } \\
\hline $\begin{array}{l}\text { Before use, thaw } \\
\text { warmed in } 37^{\circ} \mathrm{C}, 7\end{array}$ & $\begin{array}{l}20 \mathrm{~mL} \text { of SCM ( } 0.5 \% \text { lysolecithin in SCM), } \\
\text { ed, vortex to dissolve lysolecithin into SCM. }\end{array}$ \\
\hline
\end{tabular}

Table 1: List of components and protocol to make slice culture medium (SCM), dissection medium (DM), and lysolecithin solution.

\section{Discussion}

This protocol describes an organotypic cerebellar slice culture model that recapitulates in vivo cellular composition with the simplicity of an in vitro model. This protocol could be further developed to become a more representative model of human pathologies. CSC can be potentially developed as models for disease-specific injuries, such as force-induced tissue injury, injury induced by myelin-specific antibodies, or oligodendrocyte injury in MS with the addition of peripheral immune cells. This protocol can also be optimized for tissue 
dissected from other parts of the brain and spinal cord ${ }^{16}, 17$. While this protocol focuses on the histological quantification of myelination, CSC represent a convenient model for examining various experimental endpoints. These cultures can be used to characterize different cell types via single cell RNA sequencing, assess the ultrastructure of axons and myelin sheaths via electron microscopy, and study OPC dynamics by conducting time lapse imaging. Immunostaining can also be bypassed with the use of transgenic mouse reporter lines ${ }^{18,19}$. Finally, different ages of mice pups, time in culture, or thickness of sections may be used to adapt CSC to model different disease mechanisms $10,17,18,19,20,23$.

Slice cultures are ideal for testing and quantifying factors affecting myelination and remyelination but are limited in their representation of the CNS in certain contexts. Derived from $\mathrm{P} 0-2$ brains, CSC come from brains that are early in development, and have limited similarities to an aging or neurodegenerative brain. Given an increasing awareness of the importance of myelin in CNS disorders such as Alzheimer's Disease ${ }^{21}$ and schizophrenia ${ }^{22}$, adult or aging models of quantifying and characterizing myelination are required. Other protocols have been published isolating tissue from older rodents, although still during the developmental stages $10,18,19,23$. In addition, there may be intrinsic and extrinsic differences in oligodendrocytes and OPCs in the cerebellum compared to the spinal cord and other white matter tracts in the brain. CSC are also less suitable than cell-based in vitro systems for large-scale CRISPR or small molecule screens. Due to the initial tissue slicing, there is also an activation of brainresident innate immune cells (i.e., microglia and astrocytes), an important caveat to the system ${ }^{24}$. Finally, peripheral immune cells play a large role in MS pathology 25 ; CSC are devoid of peripheral cells unless these cells are exogenously added, and thus are not an ideal model of the inflammatory CNS milieu.

The dissection portion of this protocol is perhaps the most critical. Dissection steps must be undertaken with the utmost care, so as not to damage the tissue and underlying structure. Proper alignment of the brain along the tissue chopper blade ensures minimal damage to the tissue and cells. In addition, careful dissection and separation of slices, as well as their transfer to the membranes for culture are important. The timeframes outlined in this protocol have been optimized for the described research but may need to be adjusted for optimal results in different studies. This protocol will be useful for those that would like to study developmental myelination and myelin repair.

Of note, there is no cure for MS. While current therapies are quite effective at dampening the adaptive immune system, no current therapy can halt progression. A failure of remyelination and subsequent neurodegeneration is thought to underlie the progression of $\mathrm{MS}^{2}$. The presence of OPCs in chronic MS lesions suggests that the failure of myelin repair might be due to an arrest in OPC differentiation. The use of CSC opens an avenue of discovery for myelin repair therapies that may help to reverse MS progression and restore function. Myelin repair therapy can also aid recovery in patients with spinal cord injury, where demyelinated tracts inhibit locomotor function ${ }^{26}$. Therefore, the significance of CSC lies in its suitability for identifying factors that affect mammalian demyelination and remyelination in a relatively high throughput manner compared to in vivo animal models. While many studies have used primary OPCs for screening compounds affecting differentiation, generating and isolating primary OPCs requires laborious and sequential immunopanning ${ }^{27}$. In addition, cell-based 
assays do not recapitulate the diversity and interaction of cell types present in vivo. Generating CSC from mouse pups is a fast and cost-effective model for studying myelination and remyelination that does not require expensive equipment or consumables. Cerebellar slice cultures thus represent an invaluable quantitative model for recapitulating myelination ex vivo and enable drug discovery and basic science research.

\section{Disclosures}

The authors are employees of Genentech, Inc., a member of the Roche Group.

\section{Acknowledgments}

The authors would like to thank Yun-An Shen, Roxanne Kyauk, and Chris Bohlen for constructive comments and input on this article. In addition, the authors acknowledge contributions from Charles ffrench-Constant, Andrew Jarjour, Veronique Miron, and David Rowitch in early development of related methodology previously published $11,12,13$

\section{References}

1. Purves, D., Augustine, G. J., Fitzpatrick, D. Neuroscience. 2nd edition. Sunderland (MA): Sinauer Associates. Increased Conduction Velocity as a Result of Myelination (2001).

2. Dutta, R., Trapp, B. D. Mechanisms of neuronal dysfunction and degeneration in multiple sclerosis. Progress in Neurobiology. 93 (1), 1-12 (2011).

3. Trapp, B. D., Nave, K.A. Multiple sclerosis: an immune or neurodegenerative disorder? Annual Review of Neuroscience. 31, 247-269 (2008).

4. Bradl, M., Lassmann, H. Oligodendrocytes: biology and pathology. Acta Neuropathologica. 119 (1), 37-53 (2010).
5. Domingues, H. S., Portugal, C. C., Socodato, R., Relvas, J. B. Oligodendrocyte, Astrocyte, and Microglia Crosstalk in Myelin Development, Damage, and Repair. Frontiers in Cell and Developmental Biology. 4, 71 (2016).

6. Nave, K. A., Werner, H. B. Myelination of the nervous system: mechanisms and functions. Annual Review of Cell and Developmental Biology. 30, 503-533 (2014).

7. Franklin, R. J. M., Ffrench-Constant., C. Regenerating CNS myelin - from mechanisms to experimental medicines. Nature Reviews Neuroscience. 18 (12), 753-769 (2017).

8. Molina-Gonzalez, I., Miron, V. E. Astrocytes in myelination and remyelination. Neuroscience Letters. 713, 134532 (2019).

9. Keough, M. B., Jensen, S. K., Yong, V. W. Experimental demyelination and remyelination of murine spinal cord by focal injection of lysolecithin. Journal of Visualized Experiments. (97), e52679 (2015).

10. Birgbauer, E., Rao, T. S., Webb, M. Lysolecithin induces demyelination in vitro in a cerebellar slice culture system. Journal of Neuroscience Research. 78, 157-166 (2004).

11. Fancy, S. P. et al. Axin2 as regulatory and therapeutic target in newborn brain injury and remyelination. Nature Neuroscience. 14 (8), 1009-1016 (2011).

12. Yuen, T. J., et al. Identification of endothelin-2 as an inflammatory factor that promotes CNS remyelination. Brain. 136, 1035-1047 (2013).

13. Yuen, T. J. et al. Oligodendrocyte-encoded HIF function couples postnatal myelination and white matter angiogenesis. Cell. 158 (2), 383-396 (2014).

14. Stokes, C. E., Murphy, D., Paton, J. F., Kasparov, S. Dynamics of a transgene expression in acute rat brain 
slices transfected with adenoviral vectors. Experimental Physiology. 88, 459-466 (2003).

15. Dillenburg, A. et al. Activin receptors regulate the oligodendrocyte lineage in health and disease. Acta Neuropathologica. 135 (6), 887-906 (2018).

16. Dean, J. M. et al. An organotypic slice culture model of chronic white matter injury with maturation arrest of oligodendrocyte progenitors. Molecular Neurodegeneration. 6, 46 (2011).

17. Zhang, H., Jarjour, A. A., Boyd, A., Williams, A. Central nervous system remyelination in culture--a tool for multiple sclerosis research. Experimental Neurology. 230 (1), 138-148 (2011).

18. Hill, R. A., Medved, J., Patel, K. D., Nishiyama, A. Organotypic slice cultures to study oligodendrocyte dynamics and myelination. Journal of Visualized Experiments. (90), e51835 (2014).

19. Sherafat, A., Hill, R. A., Nishiyama, A. Organotypic Slice Cultures to Study Oligodendrocyte Proliferation, Fate, and Myelination. Methods in Molecular Biology (Clifton, N.J.). 1791, 145-156 (2018).

20. Croft, C. L., Noble, W. Preparation of organotypic brain slice cultures for the study of Alzheimer's disease. F1000Research. 7, 592 (2018).

21. Nasrabady, S. E., Rizvi, B., Goldman, J. E., Brickman, A. M. White matter changes in Alzheimer's disease: a focus on myelin and oligodendrocytes. Acta Neuropathologica Communications. 6 (1), 22 (2019).

22. Flynn, S. W. et al. Abnormalities of myelination in schizophrenia detected in vivo with MRI, and post-mortem with analysis of oligodendrocyte proteins. Molecular Psychiatry. 38, 811-820 (2003).
23. Thetiot, M., Ronzano, R., Aigrot, M. S., Lubetzki, C., Desmazières, A. Preparation and Immunostaining of Myelinating Organotypic Cerebellar Slice Cultures. Journal of Visualized Experiments. (145), e59163 (2019).

24. Gerlach, J., Donkels, C., Münzner, G., Haas, C. A. Persistent Gliosis Interferes with Neurogenesis in Organotypic Hippocampal Slice Cultures. Frontiers in Cellular Neuroscience. 10131 (2016).

25. Reich, D. S., Lucchinette, C. F., Calabresi, P. A. Multiple Sclerosis. New England Journal of Medicine. 378 (2), 169-180 (2018).

26. Jeffery, N. D., Blakemore, W. F. Locomotor deficits induced by experimental spinal cord demyelination are abolished by spontaneous remyelination. Brain. 120, 27-37 (1997).

27. Emery, B., Dugas, J. C. Purification of oligodendrocyte lineage cells from mouse cortices by immunopanning. Cold Spring Harbor Protocols. 854-868 (2013). 